\title{
Feedback Couplings Evaluation on Synthetic Inertia Provision for Grid Frequency Support
}

\author{
Alberto Bolzoni*, Member, IEEE, and Roberto Perini*, Member, IEEE,
}

\begin{abstract}
Emulation mechanisms of the physical synchronous generators obtained through convert-interfaced units are becoming extremely popular in literature. Still the impact of these strategies on networks frequency transients often does not take into account the effect of the converter DC-bus regulation, that plays a significant role in the dynamics definition. Thus two of the main synthetic inertia architectures are analysed both mathematically and experimentally in this paper: differently from the available literature, the goal is not just to compare their macroscopic effects on network behaviour, but rather to identify the impact of the multiple internal control feedbacks on the inertia provision itself.
\end{abstract}

Index Terms-Power systems control, Synthetic inertia, Network transients.

\section{INTRODUCTION}

$\mathbf{T}$ HE lack of inertia due to synchronous generation dismantlement is a challenge in modern power systems. A recent report from ENTSO-E [1] correlates the reduced inertia with the increasing risk of instability mechanisms in public grids and highlights the need of new regulation paradigms for converter-interfaced units able to heal these phenomena. All the available architectures share an equivalent operating principle: a local energy buffer (stored within the system or obtained keeping a margin with respect to the maximum available production) is modulated as a function of the network frequency. These techniques can be classified (Fig.1) according to the converter-control architecture and the physical nature of the energy buffer / primary source [2].

As regards the grid-supporting converters [3], the concepts of Virtual Synchronous Machine (VSM) [4] and Droop regulation [5] have been extensively analysed in literature: they are designed to contemporaneously provide inertial and steady-state regulation to the network, but they require an high energy / power ratio of the energy buffer, typically an electrochemical Battery Energy Storage.

The majority of the converter-interfaced units (e.g. renewables) are however based on the grid-following [3] scheme, typically not asked to provide regulation services to the network; however the possibility to provide some transitory grid support through this architecture has been tackled in the literature. In [6], the introduction of a regulation margin with respect to the maximum available power in a PV generator enables a transitory regulation from the unit, but involves a loss of generation due to the reserve allocation and implies a difficult estimation of the theoretical maximum power. In [7]-[8], the turbine reference angular speed in a converter-based wind generator is coupled to the network

*Dept. of Energy, Politecnico di Milano, via La Masa 34, 20156 Milano, Italy. alberto.bolzoni@polimi.it, roberto.perini@polimi.it frequency, exploiting the physical rotating mass inertia as an energy buffer: this approach does not imply any loss in the production but its applicability is limited to wind plants.

A last possibility is analysed in this paper, which consists in the provision of a transitory frequency support through the modulation of the electrostatic energy level of either a capacitive buffer [9] or an HVDC connection [10]-[11]. Due to the limited energy / power ratio, the regulation must have a transitory finite-time nature: following the nomenclature in [12], this typology goes under the name of synthetic inertia. As regards this approach, two major architectures are identified and developed in the following:

- a derivative relation between the injected power and the measured network frequency (current-controlled mode - C.C.). This architecture was initially verified by simulations in [13]-[14] and later experimentally tested for single-level [15] and multi-level converters [16], even though the impact of the DC regulation on the inertia provision has never been analysed.

- A proportional dependence between the DC bus voltage reference and the network frequency deviation (voltage-controlled mode - V.C.) is simulated in [17] and experimentally verified in [18], which provides an equivalent inertial response with no derivative calculation.

To our knowledge, a complete state-space model of the system under inertia support was never developed and compared with the experimental results: thus the novelty of this paper is the identification of a mathematical frame able to analytically, numerically and experimentally compare the synthetic inertia effects on the grid frequency transients (both for the C.C. and V.C. schemes), including the DC-bus control interaction. Section II presents the proposed complete model of the system, while simplified analytical expressions are identified in Section III to correlate the synthetic inertia impact with the DC regulation; results are verified through simulation and experimentally in Sect. IV and V respectively.

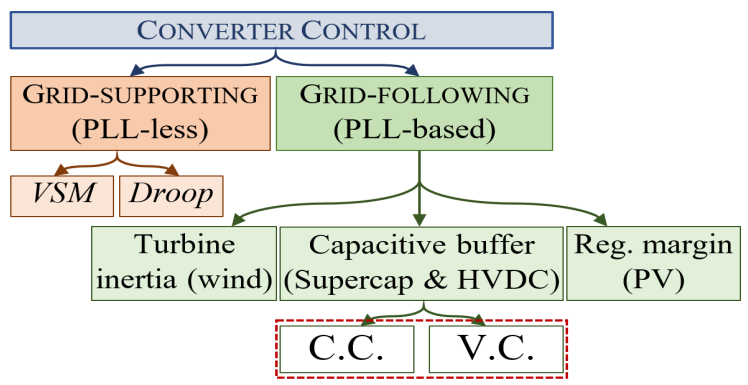

Fig. 1: Inertia provision schemes classification 


\section{NON-LINEAR MODEL OF THE SYSTEM}

\section{A. Synthetic inertia models}

Consider a grid-following converter during the provision of the two alternative synthetic inertia schemes (C.C. and V.C.) analysed in this paper (Fig. 2a). In the current-controlled (C.C.) mode, a power signal $p_{\text {in }}$ [p.u.] proportional through $K_{\text {in I }}$ (in [s]) to the estimated network angular frequency derivative $\alpha_{F L L}$ [p.u./s] is considered. For the voltage-controlled (V.C.) mode, an additional DC bus voltage reference $v_{d c \text { in }}$ [p.u.] is modulated by the estimated network frequency deviation $\Delta \omega_{F L L}$ [p.u.], proportionally to the coefficient $K_{i n V}$ (in [p.u.]). Thus (1)-(2) hold respectively:

$$
\begin{array}{llr}
\text { C.C.: } & v_{d c i n}=0 & p_{i n}=-K_{i n I} \alpha_{F L L} \\
\text { V.C.: } & p_{i n}=0 & v_{d c i n}=K_{i n V}\left(\omega_{F L L}-\omega^{*}\right)
\end{array}
$$

The estimations $\left(\omega_{F L L} / \alpha_{F L L}\right)$ of the network angular frequency $\omega$ and its derivative $\alpha$ are obtained according to [19], which can be modelled as a first order filter:

$$
p \omega_{\mathrm{FLL}}=\alpha_{\mathrm{FLL}}=\tau_{F L L}^{-1} \cdot\left(\omega-\omega_{F L L}\right)
$$

An intuitive interpretation of the proposed algorithms enables to identify their physical operating principles. Both schemes are based on the controlled discharge of an electrostatic buffer connected to the converter DC side (see Sect.I). Other approaches available in the literature propose to act on the prime energy source rather than on the electrostatic buffer [20]. Still the adopted architecture has some peculiarities with respect to other available techniques:

- it allows Maximum Power Point Operations for the prime energy source, whose injection to the DC side remains unaffected by the inertia provision; this prevents the loss of the renewable-generated energy, as the regulation-related power is exclusively provided by the local electrostatic buffer.

- The available maximum regulating energy is entirely determined by the installed buffer, rather than by the instantaneous unpredictable weather conditions (wind speed, PV solar radiation).

- The regulation shows a symmetric behaviour for over / under frequencies.

In the C.C. scheme, a power-reference $p_{i n}$ proportional to the network estimated angular frequency derivative $\alpha_{F L L}$ is introduced in the inner control loop: as the power reference definition (1) is formally equal to the state equation of a physical inertia, the converter mimics the regulation associated to a rotating mass. This scheme requires a slow DC-bus voltage regulation to allow the stored energy to be released / absorbed, before restoring the buffer to its nominal conditions.

As regards the V.C. alternative, the introduction of a frequency-dependent DC-bus voltage reference term $v_{d c i n}$ allows a controlled contribution of the electrostatic energy buffer to the network regulation [17]-[18], but it could be less intuitive to recognize that this has the same effect of a pure inertial response. A rigorous proof of this statement will be derived through the proposed mathematical model.

More in general, the goal of the paper is to provide an unequivocal mathematical representation of the converter

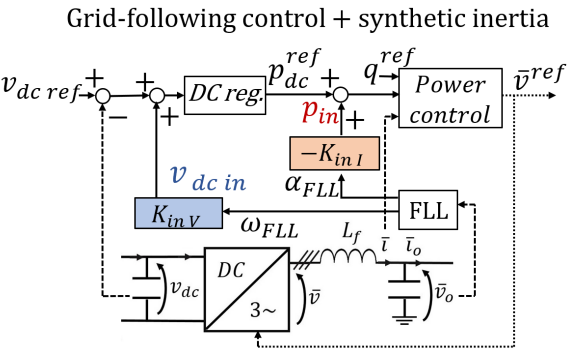

(a) Control architectures for inertia support.

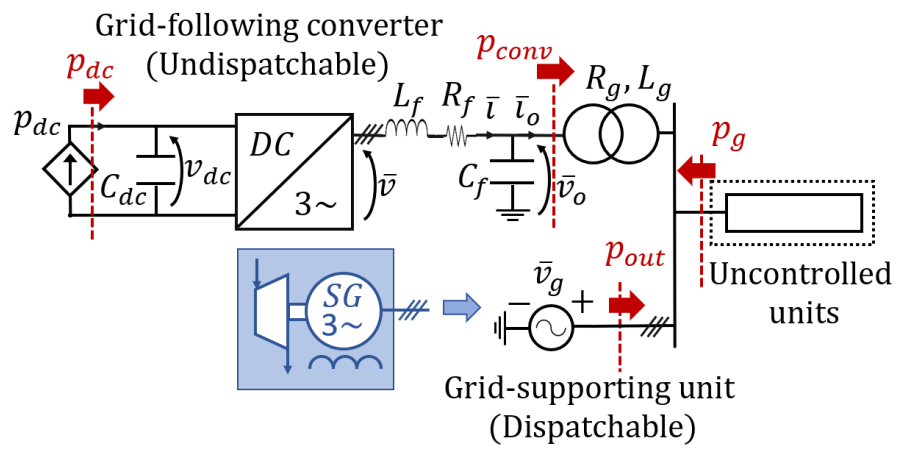

(b) Equivalent circuit of an isolated power network.

Fig. 2: (a) Alternative synthetic inertia schemes: the C.C. mode defines a power reference $p_{i n}$, while the V.C. one acts on the DC voltage $v_{d c \text { in }}$; (b) Equivalent circuit of an isolated power network, including a regulating unit, an undispatchable renewable generator (grid-following mode) and some uncontrolled local loads;

feedbacks couplings during the inertia provision and to translate the above-introduced empirical considerations on the control loops interactions in a set of formulas. To this purpose, a simplified network (Fig.2b) is introduced in the next section and later exploited as the reference-environment where to test the impact of the synthetic inertia schemes on the evolution of the grid angular frequency $\omega$ and its derivative $\alpha$.

\section{B. Grid regulation model}

Consider the per-unit instantaneous power balance (4) (neglecting losses) of the isolated network in Fig.2b (Table I), which comprises a non-dispatchable grid-following unit and a traditional dispatchable generator (this last one providing the frequency regulation): $p_{g}$ is the independent accelerating power of the network, $p_{\text {out }}$ is the contribution from regulating units and $p_{c o n v}$ is the power injected by the grid-following converter, which is typically independent of the external state of the system except when synthetic inertia is added.

$$
p_{g}+p_{\text {out }}+p_{\text {conv }}=0
$$

Under the natural variability of the accelerating power $p_{g}$, the network frequency undergoes a transient and the regulating unit modulates its output $p_{\text {out }}$ according to its physical inertia $p_{\text {inertia }}$ and primary frequency regulation $p_{\text {reg }}$.

$$
p_{\text {out }}=p_{\text {inertia }}+p_{\text {reg }}
$$

The inertia contribution $p_{\text {inertia }}$ [p.u.] can be obtained from the time derivative of the rotor kinetic energy according to the swing-equation formulation (6) [21], where $T_{a}$ is the starting 
TABLE I: Case study parameters

\begin{tabular}{|c|c|c|c|}
\hline Parameter & Symbol & Numeric value & Ref. \\
\hline \multicolumn{4}{|c|}{ Dispatchable grid-supporting regulation unit } \\
\hline Starting time & $T_{a}$ & $10 \mathrm{~s}$ & [21] \\
\hline Regulating energy & $K_{\text {reg }}$ & 50 p.u. & [22] \\
\hline Regulation delay & $\tau$ & $500 \mathrm{~ms}$ & [23] \\
\hline \multicolumn{4}{|c|}{ Non-dispatchable grid-following unit } \\
\hline AC Base voltage and power & $V_{b}, A_{b}$ & $200 \mathrm{~V}, 2.4 \mathrm{kVA}$ & \\
\hline DC Base voltage & $V_{b} d c$ & $\sqrt{2} \cdot V_{b}$ & \\
\hline Base angular frequency & $\omega_{b}$ & $2 \pi \cdot 50 \mathrm{rad} / \mathrm{s}$ & \\
\hline DC-bus capacitance & $C_{d c}$ & $8 \mathrm{mF}$ & \\
\hline Filter inductor & $R_{f}, L_{f}$ & $0.0072,0.045 \mathrm{pu}$ & \\
\hline Filter capacitor & $C_{f}$ & $0.052 \mathrm{pu}$ & \\
\hline Transformer parameters & $R_{g}, L_{g}$ & $0.037,0.012 \mathrm{pu}$ & \\
\hline Current reg. cut-off ang. freq. & $\omega_{c I}$ & $2 \pi \cdot 350 \mathrm{rad} / \mathrm{s}$ & \\
\hline Slow DC reg. cut-off ang. freq. & $\omega_{c . d c}^{\text {slow }}$ & $2 \pi \cdot 0.25 \mathrm{rad} / \mathrm{s}$ & \\
\hline $\begin{array}{l}\text { Fast DC reg. cut-off ang. freq. } \\
\text { DC reg. phase margin }\end{array}$ & $\begin{array}{l}\omega_{c d c}^{f a s t} \\
\phi_{m d c}\end{array}$ & $\begin{array}{l}2 \pi \cdot 2.5 \mathrm{rad} / \mathrm{s} \\
70 \mathrm{deg}\end{array}$ & \\
\hline FLL time constant & $\tau_{F L L}$ & $0.025 \mathrm{~s}$ & [19] \\
\hline
\end{tabular}

time of the machine and $\omega$ is the network angular frequency in [p.u.] (Table I). The symbolic operator $p=\frac{d}{d t}$ represents the time-derivative.

$$
p_{\text {inertia }}=-T_{a} \omega(p \omega)
$$

Meanwhile, the local frequency regulation acts on the primary source to compensate the network frequency $\omega$ deviation with respect to the nominal condition $\omega^{*}=1$ p.u. by modulating $p_{\text {reg }}$ according to a negative proportional law (7); the delay $\tau$ produced by the internal plant dynamics is also taken into account and expressed as a function of the derivative operator $p$ through a first-order Taylor approximation.

$$
p_{r e g}(t+\tau)=-K_{r e g} \cdot\left(\omega-\omega^{*}\right) \simeq(1+\tau p) p_{\text {reg }}(t)
$$

Substituting (7) and (6) in (5), and then again in (4), leads to express the complete grid dynamics as a second order non-linear system; the grid frequency $\omega$ and its derivative $\alpha=p \omega$ are the state variables of the system, while the network independent accelerating power $p_{g}$, its derivative $p p_{g}$ and the undispatchable converter contribution $p_{c o n v}$ are the inputs.

$$
\begin{aligned}
& \left(T_{a} \tau \omega\right) p \alpha=-K_{\text {reg }}\left(\omega-\omega^{*}\right)-T_{a} \omega \alpha-T_{a} \tau \alpha^{2}+ \\
& +\left(p_{g}+p_{\text {conv }}\right) \cdot(1+\tau p) \quad \text { where } \quad \alpha=p \omega
\end{aligned}
$$

As for the component $p_{\text {conv }}$ associated to the non-dispatchable converter (and its derivative $p p_{\text {conv }}$ ), they can be expressed as a function of the circuit state variables (voltages and currents), whose dynamics will be later highlighted.

$$
\begin{aligned}
& p_{\text {conv }}=\operatorname{Re}\left\{\bar{v}_{o d q} \bar{i}_{o d q}^{*}\right\}=v_{o d} i_{o d}+v_{o q} i_{o q} \\
& p p_{c o n v}=p v_{o d} i_{o d}+v_{o d} p i_{o d}+p v_{o q} i_{o q}+v_{o q} p i_{o q}
\end{aligned}
$$

\section{Converter dynamics and control}

The DC bus dynamic is obtained from the lossless power balance in Fig. $2 \mathrm{~b}$, where $p_{d c}$ is the instantaneous power produced by the primary source in [p.u.].

$$
\begin{aligned}
& \left(p_{d c}-R e\left[\bar{v}_{d q} \bar{i}_{d q}^{*}\right]\right) A_{b}=p\left(\frac{1}{2} C_{d c}\left(v_{d c} V_{b d c}\right)^{2}\right) \\
& p_{d c}-\left(v_{d} i_{d}+v_{q} i_{q}\right)=\tau_{d c} v_{d c} p v_{d c} \quad \tau_{d c}=\frac{C_{d c} V_{b d c}^{2}}{A_{b}}
\end{aligned}
$$

The converter AC side is modelled in the synchronous rotating frame $d q$, where $\omega$ represents the angular frequency of the external grid in [p.u.] [5]:

$$
\begin{aligned}
& p \bar{i}=\left(\bar{v}-\bar{v}_{o}-j \omega L_{f} \bar{i}-R_{f} \bar{i}\right) \omega_{b} / L_{f} \\
& p \bar{i}_{o}=\left(\bar{v}_{o}-\bar{v}_{g}-j \omega L_{g} \bar{i}_{o}-R_{g} \bar{i}_{o}\right) \omega_{b} / L_{g} \\
& p \bar{v}_{o}=\left(\bar{i}-\bar{i}_{o}-j \omega C_{f} \bar{v}_{o}\right) \omega_{b} / C_{f}
\end{aligned}
$$

The control accepts in input the reference DC bus voltage $v_{d c \text { ref }}$ and the injected reactive power reference $q^{r e f}$; the DC regulation defines the active power reference $p_{d c}^{r e f}$ according to a proportional-integral linear controller (16), where $E_{d c}$ represents the internal state variable of the voltage PI. The direct and quadrature current references are obtained from the desired injections, including the feed-forward compensation of the filter capacitor $C_{f}$ (19). Linear PI regulators are used for the DC bus voltage (16) and AC current (17):

$$
\begin{aligned}
& p_{d c}^{r e f}=\left[k_{p d c} \cdot\left(v_{d c} r e f-v_{d c}+v_{d c i n}\right)+k_{i d c} \cdot E_{d c}\right] \\
& \bar{v}^{r e f}=k_{p I} \cdot\left(\bar{i}^{r e f}-\bar{i}\right)+k_{i I} \cdot \bar{E}_{i}+\bar{v}_{o}+j \omega L_{f} \bar{i} \\
& p E_{d c}=\left(v_{d c r e f}-v_{d c}+v_{d c i n}\right) \quad p \bar{E}_{i}=\left(\bar{i}^{r e f}-\bar{i}\right) \\
& i_{d}^{r e f}=\frac{p_{d c}^{r e f}+p_{i n}}{\left|\bar{v}_{o}\right|} \quad i_{q}^{r e f}=-\frac{q^{r e f}}{\left|\bar{v}_{o}\right|}+\omega C_{f} v_{o d}
\end{aligned}
$$

Two different settings of the DC bus regulator (Table I) are considered to highlight the influence of the DC control on the service provision. To the authors' knowledge, this aspect has never been analysed in other papers dealing with the synthetic inertia provision through power converters.

\section{Eigenvalues analysis}

Figure 3 provides a graphical representation of the complete $13^{\text {th }}$-order non-linear model of the network $p \underline{x}=f(\underline{x}, \underline{u})$, obtained combining (8) - (19); state $\underline{x}$ and input $\underline{u}$ vectors are identified in (20)-(21).

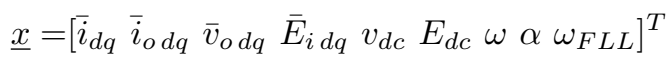

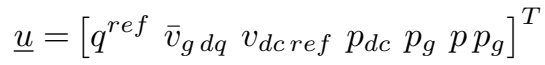

Figure 4 a shows the effect of an increasing positive inertia coefficient $K_{\text {in } I}$ on the dominant complex conjugate network eigenvalues (obtained from the numerical solution of the full order model) for the C.C. scheme, under different pass-bands of the DC control. Depending on the DC regulation settings, an increasing inertia coefficient $K_{\text {in } I}$ leads to different effects on the dominant modes: whenever $\omega_{c d c}=\omega_{c d c}^{\text {slow }}$ a markable reduction of the eigenvalues natural angular frequency is experienced and practically no effect on the damping, while a tinier impact is seen as $\omega_{c d c}=\omega_{c d c}^{f a s t}$ (with even a reduction of the complex conjugate poles damping $)^{1}$.

A similar analysis is carried out under the V.C. scheme in Fig. 4b: differently from the C.C. case, the eigenvalues damping always improves under the coefficient $K_{i n V}$ increase but the size of this change is affected by the DC bus controller.

\footnotetext{
${ }^{1}$ Eigenvalues analysis also verifies the system stability under the considered $K_{\text {in I }}$ range. Some of the transfer functions later introduced in Sect.III would also allow an analytical assessment of the stability properties, but this is out of the paper scope: a preliminary analysis is however available in [24].
} 


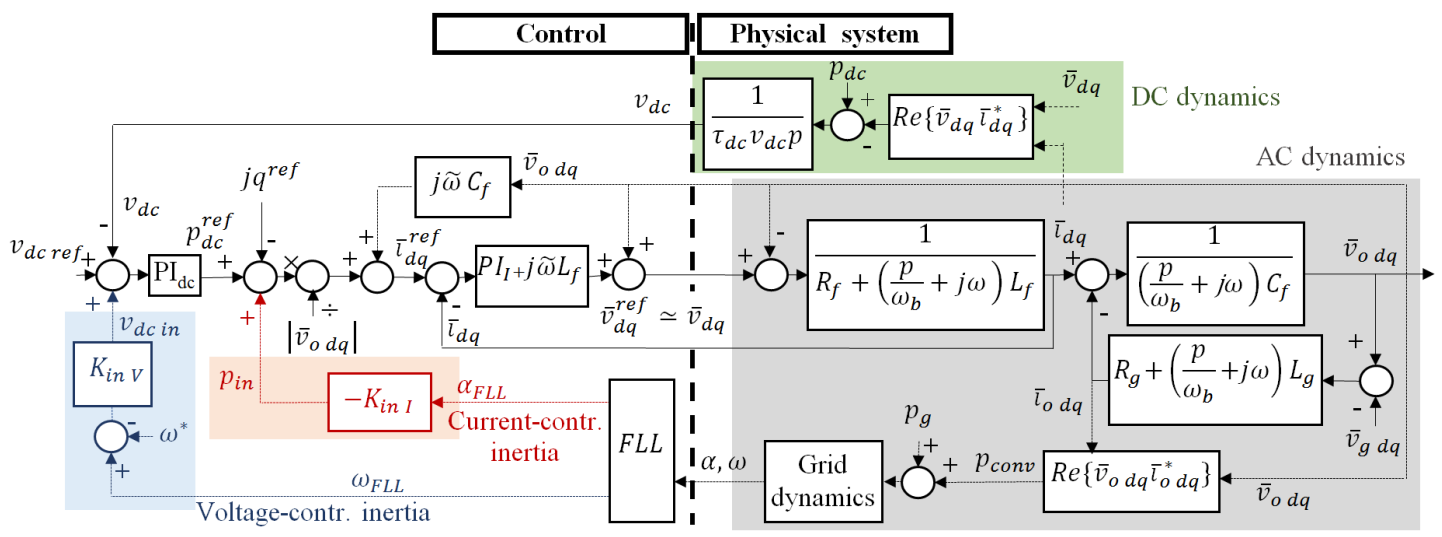

Fig. 3: Non-linear control model of the system under inertia regulation

Furthermore, depending on the DC regulation settings $\omega_{c} d c=$ $\left\{\omega_{c d c}^{\text {slow }} ; \omega_{c d c}^{f a s t}\right\}$ their natural angular frequency may increase or reduce respectively.

Fig.4 (and the full-order numerical analysis in general) allows an exact tracking of the network singularities under the C.C. or V.C. synthetic inertia introduction, but leaves space to a couple of unsolved issues. The first one deals with the possibility to predict the dominant eigenvalues evolution through simplified analytical formulas, rather than by means of the numerical full-order model. The second is associated to the identification of the damping / natural angular frequency impact on the network regulation, as both these quantities contribute to the macroscopic transient characteristics definition (sometimes counter-acting).

The paper tackles both aspects: in Sect.III, the effect of the C.C. and V.C. schemes on the damping and natural frequency of the dominant network modes is analytically derived; these results are later combined with the model in Appendix to assess the combined damping / natural frequency effects on the macroscopic network transients. The exact numerical results from the eigenvalues analysis are exploited, as a comparison, to assess the correctness of the derived analytical formulations.

\section{SIMPLIFIED LINEAR SYSTEMS}

A Laplace-based representation of system elements is derived to retrieve analytical evaluation of the inertia impact. As regards the network, the small-variation approach is applied to (8), together with some simplifying hypotheses (capital values stand for the steady-state):

- the system is initially operated in its nominal conditions, thus $\Omega=\omega^{*}=1$ p.u. and the angular frequency derivative $\alpha$ is null at steady-state. Under small-variations, it holds $\Delta \omega<\Omega$.

- Without loss of generality, the initial conditions of the system are set to $P_{g}=P_{c o n v}=0$.

- In the Taylor linearisation of (8), the steady state condition implies that $\alpha$ and $p \alpha$ are zero.

Under these hypotheses, the small variation approach applied to (8) leads to the transfer function $K_{g}(s)$ reported in (22), where $s$ is the Laplace variable: this formulation allows to link the differential frequency dynamics $\Delta \omega$ with the variation of the accelerating power $\Delta p_{g}$ and the converter

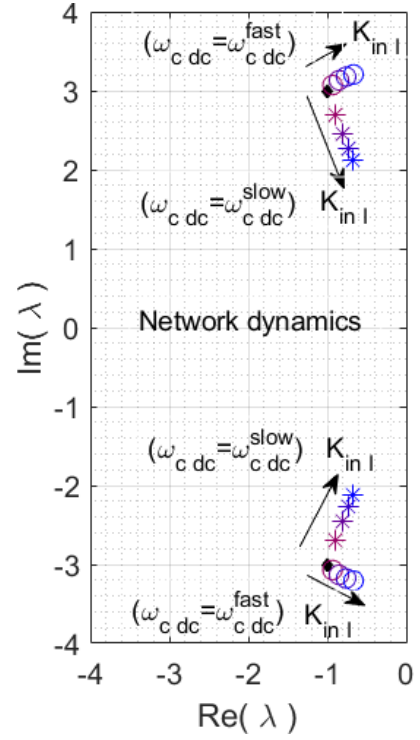

(a) C.C. mode

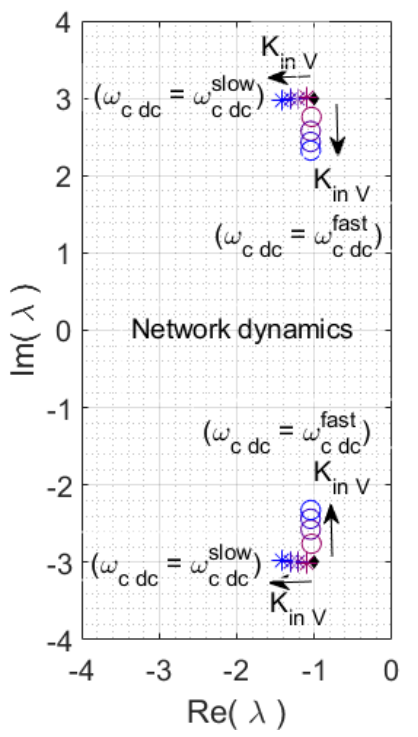

(b) V.C. mode
Fig. 4: Synthetic inertia effect on the network dynamics for (a) C.C. $\left(K_{\text {in } I} \in[0 ; 8]\right.$ p.u. $)$ and (b) V.C. mode $\left(K_{i n V} \in[0 ; 16]\right.$ p.u. $)$. Different settings of the DC-bus regulation are evaluated: $\omega_{c} d c=$ $\omega_{c d c}^{\text {slow }}$ (crosses) or $\omega_{c d c}=\omega_{c d c}^{\text {fast }}$ (circles).

response $\Delta p_{\text {conv }}$. In case the inertial support provided by the grid-following converter is null, it holds $\Delta p_{\text {conv }}=0$, while under synthetic inertia provision $\Delta p_{\text {conv }} \neq 0$.

$$
\begin{aligned}
& \frac{\Delta \omega}{\Delta p_{g}+\Delta p_{\text {conv }}}=K_{g}(s)=\frac{(1+s \tau) \mu}{\frac{s^{2}}{\omega_{n}^{2}}+2 \xi \frac{s}{\omega_{n}}+1} \\
& \text { where } \mu=K_{\text {reg }}^{-1} \quad \omega_{n}=\sqrt{\frac{K_{r e g}}{T_{a} \tau}} \quad \xi=\sqrt{\frac{T_{a}}{4 K_{r e g} \tau}}
\end{aligned}
$$

The changes introduced by the inertia schemes on $\mu^{\prime}, \xi^{\prime}, \omega_{n}^{\prime}$ are analysed in the following. Considering the values in Table I, the poles in (22) are complex conjugate.

\section{A. Current-controlled inertia}

Figure 5 shows a simplified linearised equivalent (derived from Fig. 3) of the active power balance ( $d$-component of the control) of the system under C.C. inertia. Two feedbacks 


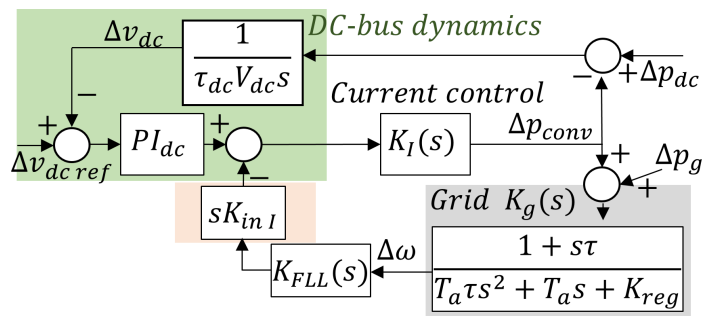

Fig. 5: Linearised model of the system for current-controlled inertia.

can be identified: the first defines the DC regulation, while the second models the converter interaction with the external grid; as the internal current control $K_{I}(s)$ and FLL estimator $K_{F L L}(s)$ show higher cut-off frequencies than the DC bus regulation and grid dynamics (Tab. I), they are approximated as unitary. The open-loop functions of the independently considered feedbacks are $L_{d c}(s)$ and $L_{i n} I(s)$ (Fig. 6), where the sign in $L_{d c}(s)$ is compensated by the negative values of the $P I_{d c}$ coefficients to produce a stable loop.

$$
L_{d c}(s)=-\frac{P I_{d c}}{\tau_{d c} V_{d c} s} \quad L_{i n I}(s)=K_{g}(s)\left(s K_{\text {in } I}\right)
$$

Simple block-diagram operations allow to express the frequency change $\Delta \omega$ as a function of the accelerating power variation $\Delta p_{g}$, leading to the modified grid function $K_{g}^{\prime}(s)$.

$$
\Delta \omega=\frac{K_{g}(s)}{1+\frac{L_{i n I}(s)}{1+L_{d c}(s)}} \Delta p_{g}=K_{g}^{\prime}(s) \Delta p_{g}
$$

The expression in (25) is general and holds independently of the relative characteristics of the DC-regulation and the synthetic inertia provision, which are expressed by the open-loop functions (24). Still it is important to analyse the possibility to predict the evolution of the grid-dynamics under different settings of the DC-bus cut-off frequency $\omega_{c d c}$ and inertia coefficient $K_{\text {in } I}$, by tracking the evolution of the corresponding poles natural frequency $\omega_{n}^{\prime}$ and damping $\xi^{\prime}$, as well as the static gain $\mu^{\prime}$ of the transfer function in (25).

The inertia impact on the static gain $\mu^{\prime}$ is obtained from the asymptotic behaviour of $L_{i n I}(s)$ and $L_{d c}(s)$ for $s \rightarrow 0$ :

$$
\mu^{\prime}=\lim _{s \rightarrow 0} K_{g}^{\prime}(s)=\lim _{s \rightarrow 0} \frac{K_{g}(s)}{1+\frac{L_{i n I}(s)}{1+L_{d c}(s)}}=\mu
$$

while the modified $\omega_{n}^{\prime}$ and $\xi^{\prime}$ are affected by both the DC regulation cut-off frequency $\omega_{c d c}$ and the grid natural angular frequency $\omega_{n}$. To reconstruct how the proposed C.C. inertia scheme acts on the network poles, it is necessary to track the denominator behaviour in (25) close to $\omega_{n}$, depending on the positions of $\omega_{c d c}$.

In case $\omega_{n}<\omega_{c d c}$ (that is: $\omega_{c d c}=\omega_{c d c}^{f a s t}$ ) the C.C. inertia impact on dominant grid modes tends to be negligible: as the DC-bus cut-off angular frequency $\omega_{c d c}$ moves toward higher frequencies, the equivalent Bode diagram of $\left|1+L_{d c}\left(j \omega_{s}\right)\right|^{-1}$ shifts downward, attenuating the inertia contribution provided by $\left|L_{i n I}\left(j \omega_{s}\right)\right|$. In an extreme case, the approximation in correspondence to $\omega_{n}$ of the combined interaction of $L_{d c}(s)$ and $L_{\text {in } I}(s)$ is expressed by the limit:

$$
\lim _{\omega_{c d c} \rightarrow+\infty}\left|\frac{L_{\text {in } I}\left(j \omega_{n}\right)}{1+L_{d c}\left(j \omega_{n}\right)}\right|=0
$$
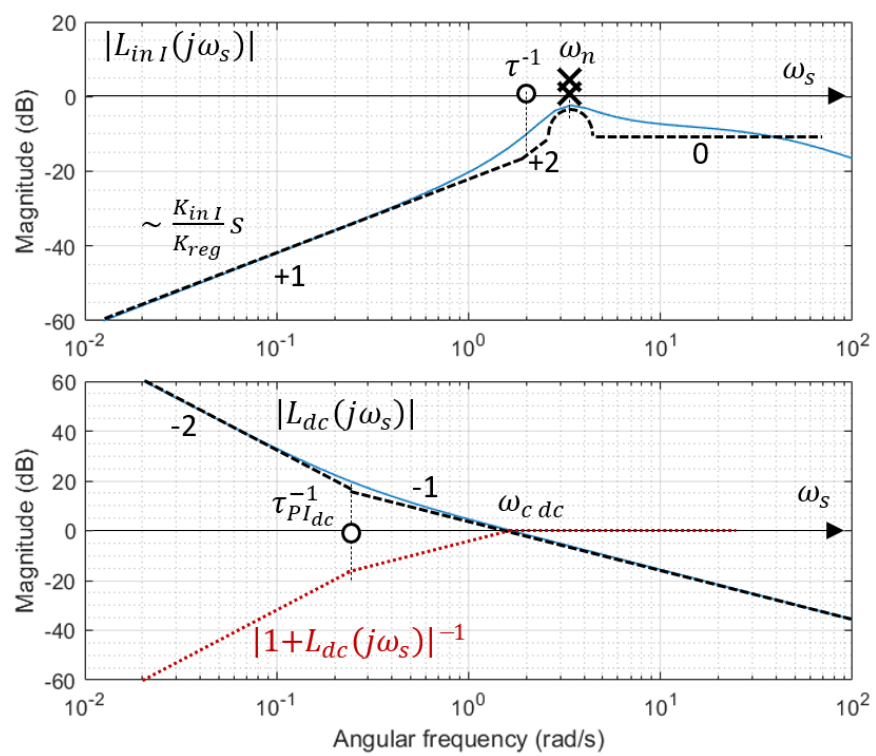

Fig. 6: Open-loop inertia function $L_{i n} I$ and DC regulation open-loop function $L_{d c}$; the asymptotic approximation of the DC feedback sensitivity $\left|1+L_{d c}(s)\right|^{-1}$ is also reported. Numeric values refer to the case $\omega_{c d c}=\omega_{c d c}^{\text {slow }}$.

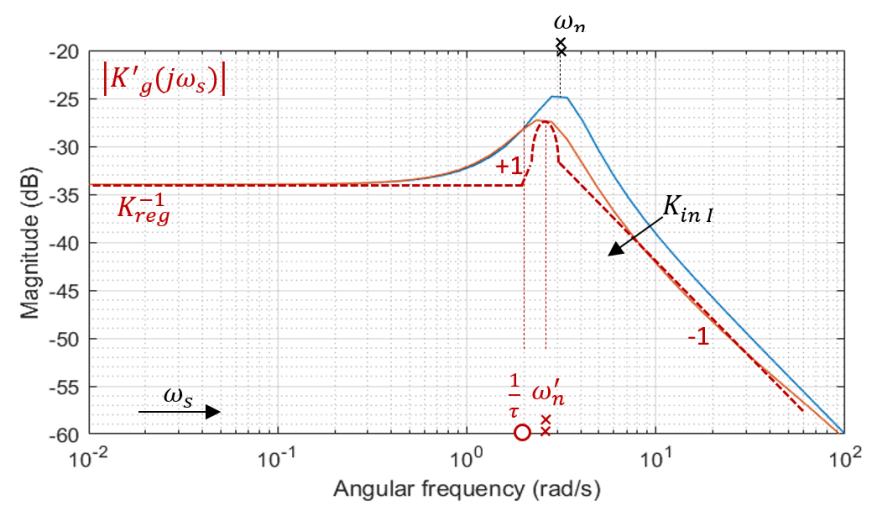

Fig. 7: Modified grid function $K_{g}^{\prime}(s)$ after inertia introduction.

Substituting (27) in (25) leads to a modified grid function $K_{g}^{\prime}(s)$ which is practically equal to the original one $K_{g}(s)$ and whose natural frequency and damping are expressed by (23). In this case, a fast DC-bus regulation jeopardizes the transitory damping provision from the C.C. synthetic inertia scheme, inhibiting an effective contribution of the converter to the network transients regulation. This result is coherent with the eigenvalues analysis in Fig.4a, which shows a weak sensitivity of the network dynamics with respect to the increasing $K_{\text {in } I}$ whenever the DC-regulation cut-off $\omega_{c d c}$ is chosen higher than the grid modes natural frequency $\omega_{n}$.

A more interesting situation occurs whenever $\omega_{n}>\omega_{c} d c$ (that is when $\omega_{c d c}=\omega_{c d c}^{\text {slow }}$ ), as the partial decoupling between the inertia and DC-regulation feedbacks enables the improvement of the network transients characteristics: whenever $\omega_{n}>\omega_{c d c}$ the open-loop DC function can be approximated as $L_{d c}(s) \simeq \omega_{c d c} / s$ in the considered range of frequency. Thus the substitution in (25) leads to a third order model expressed by (28). 


$$
\begin{aligned}
& K_{g}^{\prime}(s)=\frac{(1+s \tau)\left(s+\omega_{c d c}\right)}{s^{3} a+s^{2} b+s c+d} \\
& a=\left(T_{a} \tau+K_{\text {in I }} \tau\right) \quad b=\left(T_{a} \tau \omega_{c d c}+K_{\text {in I }}+T_{a}\right) \\
& c=\left(K_{\text {reg }}+T_{a} \omega_{c d c}\right) \quad d=K_{\text {reg }} \omega_{c d c}
\end{aligned}
$$

As $\omega_{n}>\omega_{c d c}$, (28) shows a low-frequency pole around $\omega_{c d c}$ which almost simplifies with the corresponding zero: this can be obtained performing the polynomial division of the denominator by $\left(s+\omega_{c d c}\right)$ and evaluating the remainder under the numerical values reported in Tab.I. The obtained quotient is formally equal to the one in (22), but its parameters $\xi^{\prime}-\omega_{n}^{\prime}$ (29)-(30) are affected by both inertia coefficient $K_{\text {in } I}$ and by the DC cut-off $\omega_{c d c}$ due to the partial coupling between the feedbacks (Fig. 7).

$$
\begin{aligned}
& \xi^{\prime}=\frac{\left(T_{a}+K_{\text {in } I}-K_{\text {in } I} \tau \omega_{c d c}\right)\left(\sqrt{\tau T_{a}+\tau K_{\text {in } I}}\right)^{-1}}{2 \sqrt{K_{\text {reg }}+\left(\tau \omega_{c d c}-1\right) K_{\text {in } I} \omega_{c d c}}} \\
& \omega_{n}^{\prime}=\sqrt{\frac{K_{\mathrm{reg}}+\left(\tau \omega_{c d c}-1\right) K_{\text {in } I} \omega_{c d c}}{T_{a} \tau+K_{\text {in } I} \tau}}
\end{aligned}
$$

Equations (29)-(30) are significant as they allow to analytically predict the evolution of the dominant eigenvalues in Fig.4a as a combination of the C.C. support and DC regulation; the numerical evaluation of the correspondence between the full model with the simplified formalizations (29)-(30) (as well as with the experimental test) will be performed in Sect.IV.

The model in Fig. 5 also allows to predict the impact of a step accelerating power variation $\Delta p_{g}{ }^{*}$ on the DC bus; the application of the final value theorem and the resolution of the feedbacks in Fig. 5 lead to (31), which corresponds to a null DC bus voltage change at steady state $\Delta v_{d c}^{\infty}=0$.

$$
\Delta v_{d c}^{\infty}=\lim _{s \rightarrow 0} s \frac{L_{i n I}(s)}{s \tau_{d c} V_{d c}} \frac{\left(1+L_{i n I}(s)\right)^{-1}}{1+\frac{L_{d c}(s)}{1+L_{i n} I}(s)} \frac{\Delta p_{g}{ }^{*}}{s}=0
$$

\section{B. Voltage-controlled mode}

Following a similar approach, the simplified network model under V.C. inertia is derived (Fig. 8a). The synthetic inertia interaction with the DC voltage regulation is expressed by (34) (Fig. 8b). The transfer function $K_{g}^{\prime}(s)$ between the power change $\Delta p_{g}$ and the corresponding frequency deviation $\Delta \omega$ determines the synthetic inertia effect on the grid dynamics:

$$
\begin{aligned}
& \Delta \omega=\frac{K_{g}(s)}{1+L_{i n V}(s)} \cdot \Delta p_{g}=K_{g}^{\prime}(s) \cdot \Delta p_{g} \\
& K_{d c}(s)=\left.\frac{\Delta v_{d c}}{\Delta v_{d c r e f}}\right|_{K_{i n V}=0}=\frac{L_{d c}(s)}{1+L_{d c}(s)} \simeq \\
& \simeq\left\{\begin{array}{cc}
1 & s=j \omega_{s}<j \omega_{c d c} \\
\frac{\omega_{c d c}}{s} & s=j \omega_{s}>j \omega_{c d c}
\end{array}\right. \\
& L_{i n V}(s)=\left(K_{i n V} \tau_{d c} V_{d c} s\right) \cdot K_{d c}(s) \cdot K_{g}(s)
\end{aligned}
$$

Independently of the relative position of the grid natural frequency $\omega_{n}$ and DC-bus voltage loop cut-off frequency $\omega_{c d c}$, the parameter $\mu^{\prime}$ is unaffected by the inertia introduction, leading to the same limit in (26); nevertheless, as in the C.C.

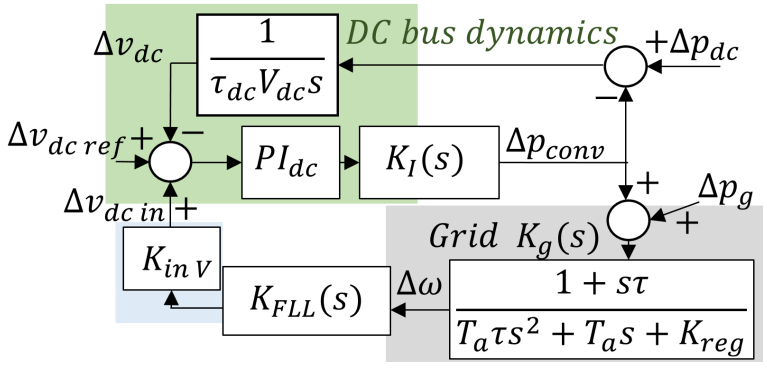

(a)

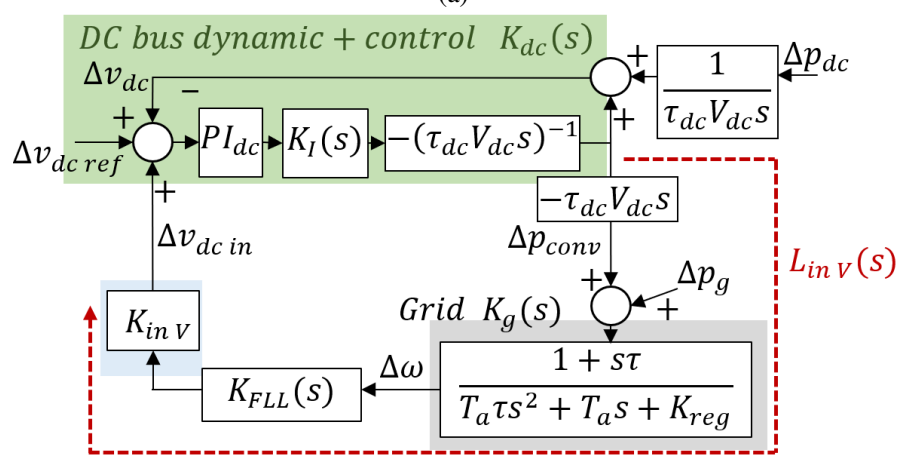

(b)

Fig. 8: (a) Linearised model for voltage-controlled inertia loop design and (b) block diagram operations.

mode, this is not the case for the grid modes natural frequency $\omega_{n}^{\prime}$ and damping $\xi^{\prime}$.

Looking at the structure of $L_{i n V}(s)$ in (34), it is possible to recognize that the maximum inertia support in correspondence to the grid dynamics location $\omega_{n}$ occurs whenever $\left|K_{d c}\left(j \omega_{n}\right)\right|=1$, that is for $\omega_{n}<\omega_{c d c}$ (e.g. $\omega_{c d c}=$ $\left.\omega_{c d c}^{\text {fast }}\right)$. The analytical substitution of the corresponding asymptotic expression (33) in (34) leads to a model formally equal to (22), but where $\omega_{n}^{\prime}$ and $\xi^{\prime}$ are set by (35) (Fig.9):

$$
\omega_{n}^{\prime}=\sqrt{\frac{K_{r e g} \tau^{-1}}{T_{a}+\tau_{d c} K_{i n V} V_{d c}}} \quad \xi^{\prime}=\sqrt{\frac{T_{a}+\tau_{d c} K_{i n V} V_{d c}}{4 K_{r e g} \tau}}
$$

This is the most interesting case as it is the one where the V.C. inertia plays a substantial role in the grid dynamics definition: the introduction of an increasing inertia coefficient $K_{\text {in } V}$ significantly improves the dynamical behaviour of the network modes, contemporaneously acting on their natural frequency $\omega_{n}^{\prime}$ and damping $\xi^{\prime}$. The expressions (35) track the eigenvalues evolution in Fig.4b when $\omega_{n}<\omega_{c d c}$ : the comparison with the full-model results will be evaluated in Sect.IV.

Similar expressions for $\omega_{n}>\omega_{c d c}$ (that is for $\omega_{c d c}=$ $\left.\omega_{c d c}^{\text {slow }}\right)$ can be also obtained, even though their importance is limited due to their reduced impact on the frequency dynamics coherently with the low sensitivity associated to the eigenvalues Fig.4b - Slow DC reg.).

$$
\begin{aligned}
& \omega_{n}^{\prime}=\sqrt{\frac{K_{r e g}+\omega_{c d c} \tau_{d c} K_{i n V} V_{d c}}{\tau T_{a}}} \\
& \xi^{\prime}=\frac{T_{a}+\omega_{c d c} \tau \tau_{d c} K_{i n V} V_{d c}}{\sqrt{4 T_{a} \tau\left(K_{\text {reg }}+\omega_{c d c} \tau_{d c} K_{i n} V_{d c}\right)}}
\end{aligned}
$$



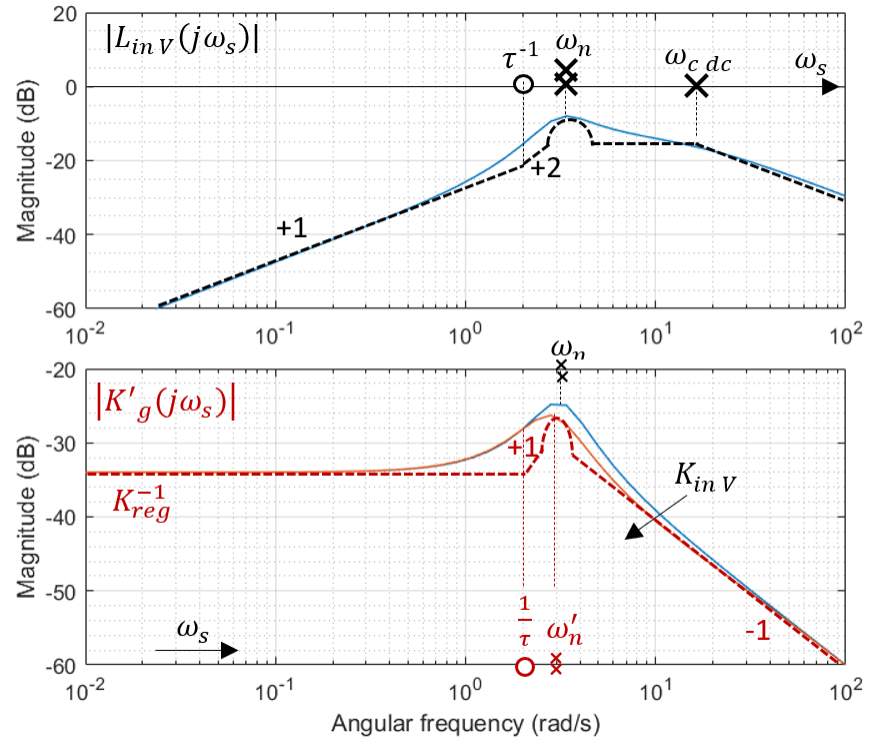

Fig. 9: Open-loop transfer function $L_{i n V}$ associated to the V.C. scheme and its modified grid function $K_{g}^{\prime}(s)$. Numeric values refer to the case $\omega_{c d c}=\omega_{c d c}^{\text {fast }}$.

It is worth noting that the DC-regulation cut-off frequency $\omega_{c d c}$ acts oppositely the C.C. and V.C. schemes: while in the first case a low cut-off (e.g. $\omega_{c d c}=\omega_{c d c}^{\text {slow }}$ ) should be preferred, the V.C. mode maximises its contribution whenever $\omega_{c d c}=\omega_{c d c}^{f a s t}$. This is coherent with the analysis in Fig.4.

The final value theorem combined with the feedbacks resolution in Fig.8a again allows to predict the DC bus steady-state condition at the end of a frequency event (38). Differently from the C.C. case, the V.C. synthetic inertia does not guarantee the DC bus voltage convergence to its initial condition after the network transient extinction, which represents a drawback of this scheme.

$$
\Delta v_{d c}^{\infty}=\lim _{s \rightarrow 0} s K_{i n V} K_{g}^{\prime}(s) K_{d c}(s) \frac{\Delta p_{g}^{*}}{s}=\frac{K_{i n V}}{K_{r e g}} \Delta p_{g}^{*}
$$

The model in Fig.8b also allows to highlight the inertial nature of the proposed architecture. The transfer function between the network angular frequency $\Delta \omega$ and the converter injection $\Delta p_{\text {conv }}$ is expressed by:

$$
\Delta p_{c o n v}=-s \tau_{d c} V_{d c} K_{i n V} K_{d c}(s) K_{F L L}(s) \Delta \omega
$$

The low-frequency behaviour can be obtained from (39), assuming $K_{d c}(s) \simeq 1$ (33) and $K_{F L L}(s) \simeq 1$, coherently with the previously introduced hypotheses. Thus:

$$
\Delta p_{\text {conv }} \sim-s \tau_{d c} V_{d c} K_{i n V} \Delta \omega
$$

which, in the time domain, corresponds to a derivative dependence of the converter injection $\Delta p_{\text {conv }}$ with respect to the frequency deviation $\Delta \omega$, formally equal to the one of physical inertial systems e.g. (6).

\section{SimUlation RESULTS}

The network in Fig.2b has been simulated through MATLAB-Simulink to test the impact of the DC-bus voltage regulation cut-off frequency $\omega_{c d c}$ on the synthetic inertia provision: the analysis shows the network transients modifications due to the provision of C.C. / V.C. synthetic inertia during a constant-power load connection $\Delta p_{g}=$ $-0.5 \mathrm{pu}$, under different setting of the grid-following converter

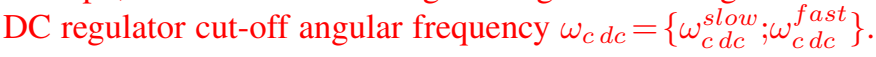

\section{A. Current-controlled inertia: simulations}

Fig. 10 shows the effect on the C.C. architecture, with a constant $K_{i n I}=6 s$ : whenever a slow voltage regulator is adopted $\omega_{c d c}=\omega_{c d c}^{s l o w}$, the C.C. synthetic inertia positively improves the network transitory behaviour, thanks to the reduction of the eigenvalues natural angular frequency observed in Fig.4a and correctly tracked by (30). This macroscopically translates into an increase of the oscillation period and an overshoot reduction with respect to the base case (Table II). This trend is verified by all the considered models: eigenvalues, simplified expressions (29)-(30) and simulations.

On the contrary, a fast DC controller $\left(\omega_{c d c}=\omega_{c d c}^{\text {fast }}\right)$ jeopardizes the service provision and degrades the network regulation performances leading to a reduction of the oscillation period and increase of the overshoot, coherently with the eigenvalue model in Fig.4a.

\section{B. Voltage-controlled inertia: simulation}

The V.C. synthetic inertia effects under different settings of the DC-bus voltage regulator are reported in Fig.11 and Table III. Coherently with the eigenvalue model (Fig.4a), both the DC regulator design conditions lead to the network frequency transients improvements but, differently from the C.C. case, this effect is maximized for $\omega_{c d c}>\omega_{n}$ : referring to the case $\omega_{c d c}=\omega_{c d c}^{f a s t}$ in Tab.III, it is possible to observe a significant reduction of the frequency overshoot and an increase of the oscillation period with respect to the base case with no synthetic inertia. On the other hand, when $\omega_{c d c}=\omega_{c d c}^{\text {slow }}$ the obtainable improvements are reduced.

It is worth highlighting the significantly-small mismatch experienced between the eigenvalue model, the approximated formulas derived in Sect.III and the simulation results: this proves the correctness of the equations (35) (for $\omega_{c d c}=\omega_{c d c}^{\text {fast }}$ ) and (36)-(37) (for $\omega_{c d c}=\omega_{c d c}^{\text {slow }}$ ).

\section{EXPERIMENTAL VALIDATION}

The verification of synthetic inertia properties has been carried out in a small-scale island operated microgrid, supplied by two independent stand-alone converters (Fig. 12). The first unit is controlled to emulate the physical behaviour of the primary regulation: it imposes an angular frequency $\omega$ transient according to (22), which depends on the dynamical characteristics of the grid regulation $\left(T_{a}, K_{\text {reg }}, \tau\right)$ and the power exchanged at its terminal. The behaviour is obtained through the bilinear-discretization of (22), where the accelerating power $p_{g}$ change is set as a parameter inside the control, while the response $p_{\text {conv }}$ from grid-following unit is measured at the physical interface (Point of Coupling - PC) ${ }^{2}$.

${ }^{2}$ Due to the unidirectional rectifier associated to the grid-forming unit, over-frequency transients only are experimentally tested to avoid over-voltages on the grid-forming unit DC bus; this does not affect the derived analytical model generality. 

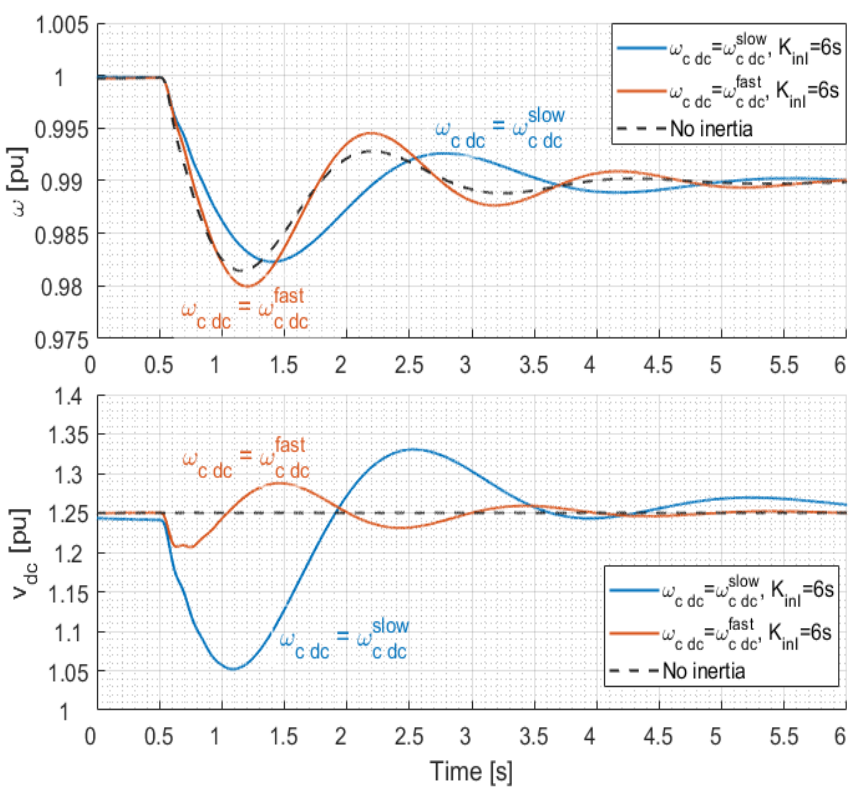

Fig. 10: Grid angular frequency $\omega$ and DC-bus voltage $v_{d c}$ under different cut-off frequencies of the DC-bus regulator with C.C. synthetic inertia coefficient $K_{i n I}=6 \mathrm{~s}$.
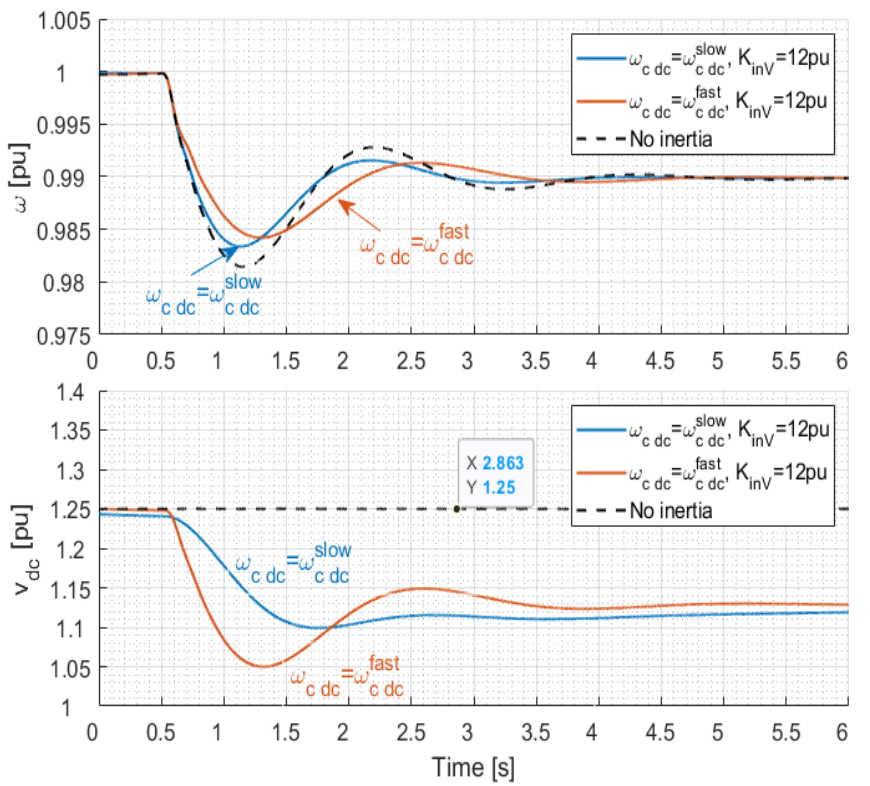

Fig. 11: Grid angular frequency $\omega$ and DC-bus voltage $v_{d c}$ under different cut-off frequencies of the DC-bus regulator with V.C. synthetic inertia coefficient $K_{i n V}=16 p u$.

The second converter is operated according to the grid-following control scheme illustrated in Fig. 3, providing synthetic inertia support to the network by modulating its injection $p_{\text {conv }}$ (C.C. or V.C. modes) under a measured transient of $\omega$.

\section{A. Current-controlled inertia: experimental results}

Figures 13 depicts the transitory time profiles of the network angular frequency and its derivative, DC bus voltage and injection $p_{c o n v}$ of the grid-following unit equipped with the synthetic inertia during an equivalent load change $\Delta p_{g}=$ $1 p . u$., with different values of the C.C. inertia coefficient
TABLE II: Current-controlled inertia - Simulations

\begin{tabular}{ccccc}
\hline Parameter & Condition & $\begin{array}{c}\text { Eigen. } \\
\text { model }\end{array}$ & $\begin{array}{c}\text { Approx. } \\
\text { formula }\end{array}$ & $\begin{array}{c}\text { Simul. } \\
\text { result }\end{array}$ \\
\hline Oscil. period & $\omega_{c \text { scow }}^{\text {slow }}, K_{\text {inI }}=6 \mathrm{~s}$ & $2.72 \mathrm{~s}$ & $2.68 \mathrm{~s}$ & $2.64 \mathrm{~s}$ \\
$T$ (eq.44) & $\omega_{c \text { fast }}^{\text {fas }}, K_{\text {inI }}=6 \mathrm{~s}$ & $1.99 \mathrm{~s}$ & $2.09 \mathrm{~s}$ & $1.98 \mathrm{~s}$ \\
& No synt. inertia & $2.09 \mathrm{~s}$ & $2.09 \mathrm{~s}$ & $2.09 \mathrm{~s}$ \\
& & & & \\
Frequency & $\omega_{c \text { sc }}^{\text {slow }}, K_{\text {inI }}=6 \mathrm{~s}$ & $69 \%$ & $69 \%$ & $77 \%$ \\
overshoot & $\omega_{c \text { fdc }}^{\text {fast }}, K_{\text {inI }}=6 \mathrm{~s}$ & $99 \%$ & $80 \%$ & $100 \%$ \\
OS (eq.45) & No synt. inertia & $80 \%$ & $80 \%$ & $84 \%$ \\
\hline
\end{tabular}

TABLE III: Voltage-controlled inertia - Simulations

\begin{tabular}{|c|c|c|c|c|}
\hline Parameter & Condition & $\begin{array}{l}\text { Eigen. } \\
\text { model }\end{array}$ & $\begin{array}{l}\text { Approx. } \\
\text { formula }\end{array}$ & $\begin{array}{l}\text { Simul. } \\
\text { result }\end{array}$ \\
\hline Oscil. period & $\omega_{c d c}^{\text {slow }}, K_{\text {in } V}=12 \mathrm{pu}$ & $2.08 \mathrm{~s}$ & $2.04 \mathrm{~s}$ & $2.07 \mathrm{~s}$ \\
\hline$T$ (eq.44) & $\begin{array}{l}\omega_{c d c}^{f a s t}, K_{i n V}=12 \mathrm{pu} \\
\quad \text { No synt. inertia }\end{array}$ & $\begin{array}{l}2.55 \mathrm{~s} \\
2.09 \mathrm{~s}\end{array}$ & $\begin{array}{l}2.66 \mathrm{~s} \\
2.09 \mathrm{~s}\end{array}$ & $\begin{array}{l}2.70 \mathrm{~s} \\
2.09 \mathrm{~s}\end{array}$ \\
\hline Frequency & $\omega_{c_{c} d c}^{\text {slow }}, K_{\text {in } V}=12 \mathrm{pu}$ & $65 \%$ & $69 \%$ & $67 \%$ \\
\hline $\begin{array}{l}\text { overshoot } \\
O S(\text { eq. } 45)\end{array}$ & $\begin{array}{l}\omega_{c d c}^{\text {fast }}, K_{i n V}=12 \mathrm{pu} \\
\quad \text { No synt. inertia }\end{array}$ & $\begin{array}{l}50 \% \\
80 \%\end{array}$ & $\begin{array}{l}54 \% \\
80 \%\end{array}$ & $\begin{array}{l}58 \% \\
84 \%\end{array}$ \\
\hline
\end{tabular}

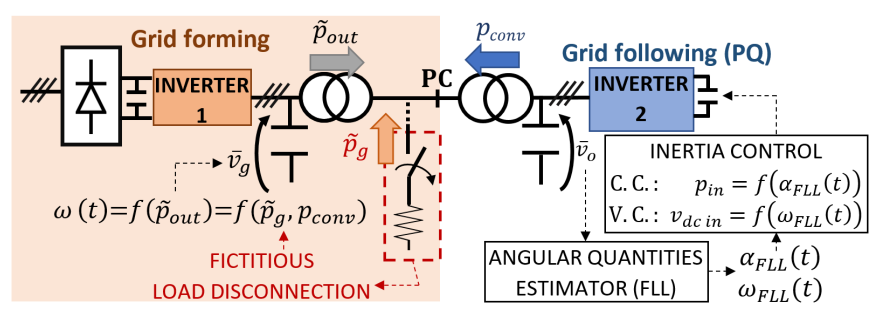

Fig. 12: Structure of the experimental set-up

$K_{\text {in I }}$ (with $\omega_{c d c}=\omega_{c d c}^{\text {slow }}$ ). The inertia introduction leads to a reduction of the frequency overshoot and Rate of Change of Frequency $R O C O F$ along the first rise. Coherently with its inertial nature, the proposed control architecture guarantees the injected power $p_{\text {conv }}$ convergence to its initial pre-transient value once the oscillation is expired, without affecting the system steady-state behaviour; the DC-bus voltage also recovers its initial state once the transient has ceased (31).

A more numerically-consistent comparison between the complete eigenvalues model, the simplified SISO representation and the experimentally-measured transients is provided: the expected oscillation period $T$, overshoot $O S$ and Rate of Change of Frequency ROCOF along the first rise are included in Table IV (see Appendix for the analytical derivation of $T, O S$ and $R O C O F$ ). The analysis shows a strong matching of the simplified SISO formulation (Fig.5) with the expected dynamics from the complete system. As regards the experimental values, a practically-perfect matching is experienced as regards the oscillation period $T$ and overshoot $O S$, while a higher deviation is associated to the $R O C O F$, as a consequence of the non-ideal estimation of the angular quantities: nevertheless, the improved transitory behaviour and the correspondence between theoretical, simplified and experimental characteristics verify the correctness of the proposed dynamical formulation. 

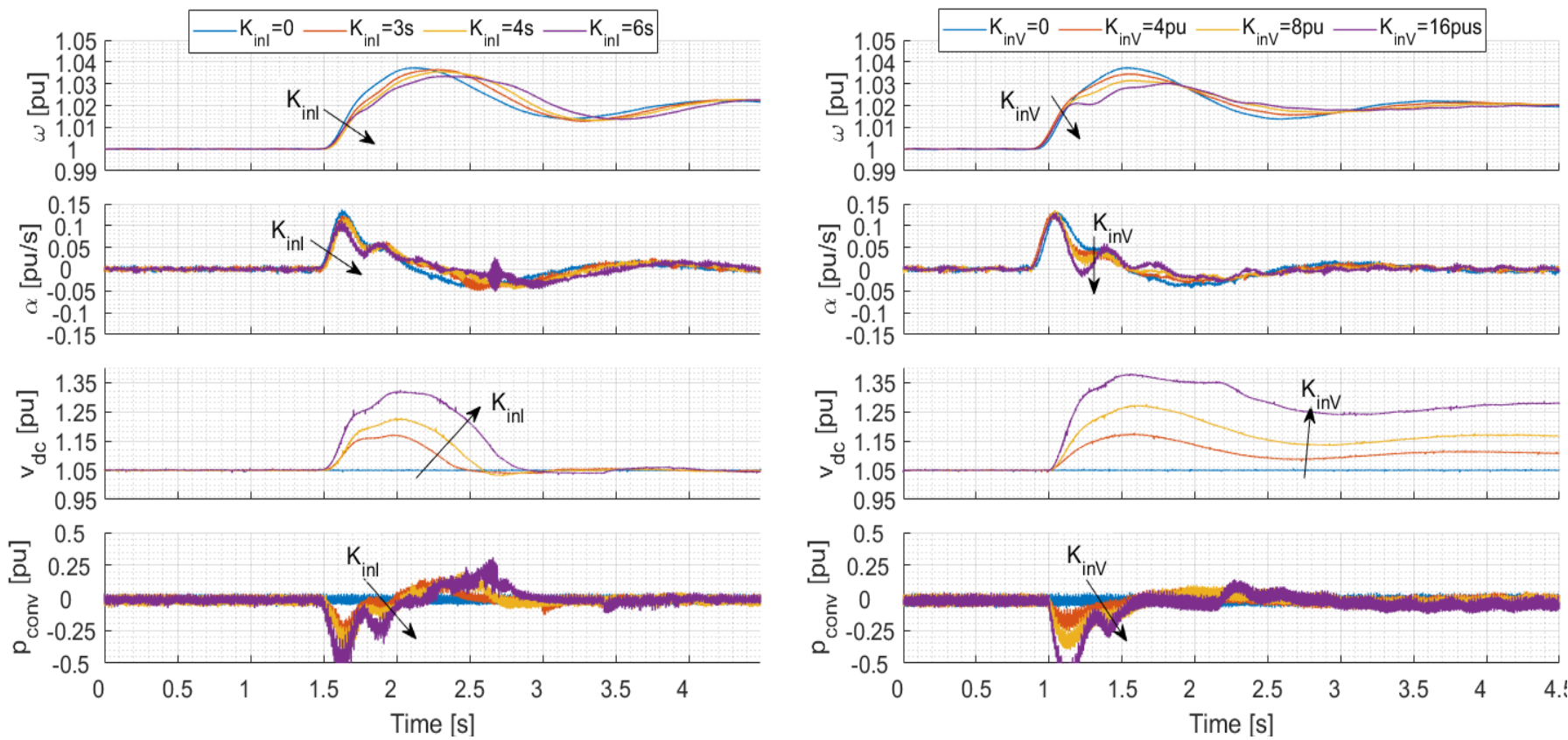

Fig. 13: Time profiles of the measured angular frequency $\omega$, its derivative alpha, DC-bus voltage $v_{d c}$ and power $p_{c o n v}$ under C.C. inertia; the overshoot reduction depends on the influence of the inertia feedback with the DC regulation.

TABLE IV: Current-controlled inertia - Experimental validation

\begin{tabular}{ccccc}
\hline $\begin{array}{c}K_{\text {in } I} \\
{[s]}\end{array}$ & Parameter & $\begin{array}{c}\text { Eigen. } \\
\text { model }\end{array}$ & $\begin{array}{c}\text { Approx. } \\
(\text { Error \%) }\end{array}$ & $\begin{array}{c}\text { Experim. } \\
\text { (Error \%) }\end{array}$ \\
\hline 0 & Period of & 2.09 & $2.09(<1 \%)$ & $2.05(2 \%)$ \\
3 & oscillation & 2.42 & $2.40(1 \%)$ & $2.07(15 \%)$ \\
4 & $T$ - eq.(44) & 2.53 & $2.49(2 \%)$ & $2.17(14 \%)$ \\
6 & [s] & 2.72 & $2.68(2 \%)$ & $2.41(12 \%)$ \\
& & & & \\
0 & Frequency & $80 \%$ & $80 \%(<1 \%)$ & $85 \%(7 \%)$ \\
3 & overshoot & $72 \%$ & $73 \%(1 \%)$ & $80 \%(10 \%)$ \\
4 & OS - eq.(45) & $71 \%$ & $72 \%(1 \%)$ & $75 \%(6 \%)$ \\
6 & [\%] & $69 \%$ & $69 \%(<1 \%)$ & $65 \%(5 \%)$ \\
& & & & \\
0 & Freq. Rate & 0.049 & $0.049(<1 \%)$ & $0.059(20 \%)$ \\
3 & of Change & 0.039 & $0.040(2 \%)$ & $0.046(20 \%)$ \\
4 & ROCOF - eq. $(44)$ & 0.036 & $0.037(3 \%)$ & $0.042(16 \%)$ \\
6 & [pu/s] & 0.033 & $0.034(3 \%)$ & $0.037(12 \%)$ \\
\hline \multicolumn{7}{c}{}
\end{tabular}

\section{B. Voltage-controlled inertia: experimental results}

The network frequency profile, frequency derivative, DC voltage and converter power $p_{\text {conv }}$ for the V.C. scheme are reported in Fig. 14, under the fast regulation settings for the DC controller. Similarly to the previous case, the converter-control scheme allows to emulate the physical inertial behaviour during a frequency transient (see the relative shapes of $\alpha$ and $p_{\text {conv }}$ ), providing transitory damping to the network without affecting its steady-state condition. Nevertheless, in this case the DC voltage converges to a non-nominal steady-state value (38), which represents a major difference with respect to the C.C. approach.

Table V shows the comparison between full-model eigenvalues, simplified expressions and experimental transients in terms of the expected overshoot $O S$, oscillation period $T$ and ROCOF (see Appendix). The limited mismatch of the simplified expressions with respect to the full-model case

Fig. 14: Time profiles of the measured angular frequency $\omega$, its derivative alpha, DC-bus voltage $v_{d c}$ and power $p_{c o n v}$ under V.C. inertia; differently from the C.C. case, the DC voltage does not reach its nominal initial condition after the transient has expired.

TABLE V: Voltage-controlled inertia - Experimental validation

\begin{tabular}{ccccc}
\hline $\begin{array}{c}K_{i n V} V \\
{[p u]}\end{array}$ & Parameter & $\begin{array}{c}\text { Eigen. } \\
\text { model }\end{array}$ & $\begin{array}{c}\text { Approx. } \\
(\text { Error \%) }\end{array}$ & $\begin{array}{c}\text { Experim. } \\
(\text { Error \%) }\end{array}$ \\
\hline 0 & Period of & 2.09 & $2.09(<\%)$ & $2.05(2 \%)$ \\
4 & oscillation & 2.27 & $2.23(2 \%)$ & $2.34(3 \%)$ \\
8 & $T$ - eq.(44) & 2.45 & $2.38(3 \%)$ & $2.62(7 \%)$ \\
16 & {$[$ [s] } & 2.88 & $2.72(5 \%)$ & $2.78(3 \%)$ \\
& & & & \\
0 & Frequency & $80 \%$ & $80 \%(<1 \%)$ & $85 \%(7 \%)$ \\
4 & overshoot & $67 \%$ & $71 \%(4 \%)$ & $70 \%(4 \%)$ \\
8 & OS - eq.(45) & $57 \%$ & $63 \%(9 \%)$ & $57 \%(1 \%)$ \\
16 & [\%] & $42 \%$ & $48 \%(15 \%)$ & $49 \%(17 \%)$ \\
& & & & \\
0 & Freq. Rate & 0.049 & $0.049(<\%)$ & $0.059(20 \%)$ \\
4 & of Change & 0.041 & $0.043(5 \%)$ & $0.050(17 \%)$ \\
8 & ROCOF - eq. $(44)$ & 0.035 & $0.037(6 \%)$ & $0.042(20 \%)$ \\
16 & [pu/s] & 0.026 & $0.028(8 \%)$ & $0.033(22 \%)$ \\
\hline \multicolumn{4}{c}{}
\end{tabular}

confirms the correctness of the derived formalism even under partial coupling of the internal feedbacks within the control.

\section{CONCLUSION}

In this paper, the dynamical models of two alternative synthetic inertia architectures have been developed: the analysis has focused not only on the impact of the proposed schemes on the network frequency transients, but rather on the dynamical coupling of the different loops within the control.

To this purpose, simplified analytical expressions which link the network frequency regulation characteristics to the converter control parameters have been derived. Their correctness is verified comparing the obtained results with the ones from experimental measurements, simulations and from a full-order eigenvalue model, reaching a very good consistency.

The analysis proves that the current-controlled scheme works better whenever the DC-bus voltage regulator has an 
equivalent cut-off frequency lower than the network regulation natural frequency; the opposite holds for the voltage-controlled scheme. Thus, depending on the dynamical characteristics of the grid, it is possible to design the control parameters of the chosen synthetic inertia architecture which maximise the service provision effectiveness.

\section{APPENDIX}

\section{EXPECTED NETWORK FREQUENCY DYNAMICS}

The expected characteristics of the network frequency evolution under the regulation (22) are calculated and exploited to evaluate the correspondence of the synthetic inertia models to the theoretical ones under a step change of the accelerating input power $\Delta p_{g}=\frac{\Delta p_{g}^{*}}{s}$ (41). The static gain $\mu^{\prime}$, the natural frequency $\omega_{n}^{\prime}$ and the damping $\xi^{\prime}$ are function of the physical network characteristics modified by the C.C. inertia as in (29)-(30), or (35) for the V.C. one. The inverse Laplace transform of (41) is obtained from the Heaviside procedure (42).

$$
\begin{aligned}
& \Delta \omega(t)=\mathcal{L}^{-1}\left[\frac{(1+s \tau) \mu^{\prime} \omega_{n}^{\prime 2}}{s^{2}+2 \xi^{\prime} \omega_{n}^{\prime} s+\omega_{n}^{\prime 2}} \cdot \frac{\Delta p_{g}^{*}}{s}\right] \\
& \Delta \omega=\mu^{\prime} \Delta p_{g}^{*}\left[1+A e^{-\xi^{\prime} \omega_{n}^{\prime} t} \sin \left(\sqrt{1-\xi^{\prime 2}} \omega_{n}^{\prime} t-\varphi_{A}\right)\right] \\
& A=\sqrt{\frac{\tau^{2} \omega_{n}^{\prime 2}-2 \xi^{\prime} \omega_{n}^{\prime} \tau+1}{1-\xi^{\prime 2}}} \quad \varphi_{A}=\operatorname{atan}\left(\frac{\sqrt{1-\xi^{\prime 2}}}{\tau \omega_{n}^{\prime}-\xi^{\prime}}\right)
\end{aligned}
$$

The free-response of (42) shows a damped oscillation with period $T$ (44) and overshoot $O S$, obtained combining $A$ and $e^{-\xi^{\prime} \omega_{n}^{\prime} t^{*}}$, being $t^{*}$ the time-instant where the sine is maximum (45); the average ROCOF during the first rise is also determined (44). These theoretical expressions are used for the C.C./V.C. inertia models validation in Tab. IV-V.

$$
\begin{aligned}
& T=\frac{2 \pi}{\sqrt{1-\xi^{\prime 2}} \omega_{n}^{\prime}} \quad \text { ROCOF }=\mu^{\prime} \Delta p_{g}^{*} \frac{1+O S}{t^{*}} \\
& O S=A e^{-\xi^{\prime} \omega_{n}^{\prime} t^{*}} \quad t^{*}=\frac{\omega_{n}^{\prime-1}\left(\frac{\pi}{2}+\varphi_{A}\right)}{\sqrt{1-\xi^{\prime 2}}}
\end{aligned}
$$

\section{REFERENCES}

[1] ENTSO-E, Guidance document for National Implement. of Network Codes, Need for synthetic inertia for frequency regulation, 2016.

[2] J. Fang, H. Li, Y. Tang, and F. Blaabjerg, "On the inertia of future more-electronics power systems," IEEE Journal of Emerging and Selected Topics in Power Electr., vol. 7, pp. 2130-2146, Dec 2019.

[3] R. Teodorescu, M. Liserre, and P. Rodriguez, Grid converters for photovoltaic and wind power systems. Wiley and Sons, 2010.

[4] U. Bose, S. K. Chattopadhyay, C. Chakraborty, and B. Pal, "A novel method of frequency regulation in microgrid," IEEE Trans. on Industry Applications, vol. 55, pp. 111-121, Jan 2019.

[5] A. Bolzoni, G. M. Foglia, L. Frosio, M. F. Iacchetti, and R. Perini, "Impact of line and control parameters on droop stability in inverters for distributed generation," IEEE Trans. on Smart Grid, vol. 9, 2018.

[6] S. Nanou, A. Papakonstantinou, and S. Papathanassiou, "A generic model of two-stage grid-connected PV systems with primary freq. resp. and inertia emulation," Electric Power Syst. Research, vol. 127, 2015.

[7] A. Bonfiglio, M. Invernizzi, A. Labella, and R. Procopio, "Design and implementation of a variable synthetic inertia controller for wind turbine generators," IEEE Trans. on Power Syst., vol. 34, pp. 754-764, Jan 2019.

[8] W. He, X. Yuan, and J. Hu, "Inertia provision and estim. of PLL-based DFIG wind turbines," IEEE Trans. on Power Syst., vol. 32, 2017.
[9] Y. Li, Z. Zhang, Y. Yang, Y. Li, H. Chen, and Z. Xu, "Coordinated control of wind farm and VSC-HVDC system using capacitor energy and kinetic energy to improve inertia level of power systems," Int'nal journal of Electic. Power and Energy Syst., vol. 59, 2014.

[10] M. Zhang, X. Yuan, and J. Hu, "Inertia and primary frequency provisions of PLL-synchronized VSC HVDC when attached to islanded AC system," IEEE Trans. on Power Syst., vol. 33, 2018.

[11] X. Liu and A. Lindemann, "Control of vsc-hvdc connected offshore windfarms for providing synthetic inertia," IEEE Journal of Emerging and Selected Topics in Power Electr., vol. 6, pp. 1407-1417, Sep. 2018.

[12] R. Eriksson, N. Modig, and K. Elkington, "Synthetic inertia versus fast frequency response: a definition," IET Renewable Power Generation, vol. 12 , no. 5 , pp. 507-514, 2018.

[13] E. Rakhshani and P. Rodriguez, "Inertia emulation in ac/dc interconnected power systems using derivative technique considering frequency measurement effects," IEEE Trans. on Power Systems, vol. 32 pp. 3338-3351, Sep. 2017.

[14] D. Duckwitz and B. Fischer, "Modeling and design of $d f / d t$-based inertia control for power converters," IEEE Journal of Emerging and Selected Topics in Power Electr., vol. 5, pp. 1553-1564, Dec 2017.

[15] J. Fang, R. Zhang, H. Li, and Y. Tang, "Frequency derivative-based inertia enhancement by grid-connected power converters with a frequency-locked-loop," IEEE Trans. on Smart Grid, 2018.

[16] S. Yang, J. Fang, Y. Tang, H. Qiu, C. Dong, and P. Wang, "Modular multilevel converter synthetic inertia-based frequency support for mv microgrids," IEEE Trans. on Ind. Electr., vol. 66, Nov 2019.

[17] J. Zhu, J. Hu, W. Hung, C. Wang, X. Zhang, S. Bu, Q. Li, H. Urdal, and C. D. Booth, "Synthetic inertia control strategy for doubly fed induction generator wind turbine generators using lithium-ion supercapacitors," IEEE Trans. on Energy Conversion, vol. 33, pp. 773-783, June 2018.

[18] J. Fang, H. Li, Y. Tang, and F. Blaabjerg, "Distributed power system virtual inertia implemented by grid-connected power converters," IEEE Trans. on Power Electronics, vol. 33, 2018.

[19] A. Bolzoni, R. Perini, "Experimental validation of novel angular estimator for synthetic inertia support under disturbed network conditions," in 21 Eur. Conf. on Power Elect. Applic. EPE ECCE, 2019.

[20] Dreidy, M. and Mokhlis, H. and Mekhilef, S., "Inertia response and frequency control techniques for renewable energy sources: A review," Renewable and Sustainable Energy Reviews, vol. 69, pp. 144-155, 2017.

[21] P. Kundur, Power system stability and control. Mc. Graw Hill, 1993.

[22] Terna (Italian TSO), Grid code attachm. A15 - Technical requirements for frequency regulation, 2008. Available at: www.terna.it.

[23] National Grid (UK TSO), Testing guidance for Enhanced Frequency Response Balancing service, 2016. Available at: www.nationalgrid.com.

[24] A. Bolzoni, C. Terlizzi, and R. Perini, "Analytical design and modelling of power converters equipped with synthetic inertia control," in 20th European Conf. on Power Electr. and Applic. EPE ECCE, 2018.

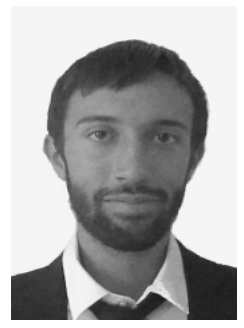

Alberto Bolzoni (M'19) received his MS and $\mathrm{PhD}$ degree in electrical engineering at Politecnico di Milano, Milano, Italy, in 2015 and 2019 respectively. Currently, he is a post-doctoral researcher at The University of Manchester, UK. His main field of interest is power electronics applied to microgrids and hybrid power plants.

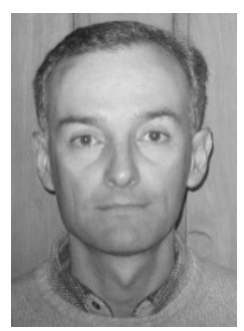

Roberto Perini (M'10) received his MS degree and the $\mathrm{PhD}$ in electrical engineering from the Politecnico di Milano, Milano, Italy. Currently, he is an Associate Professor at the Department of Energy at Politecnico di Milano. His interests are in the design and modeling of electrical machines and microgrids. 